\title{
Ordinal measurement of autistic behavior: A preliminary report
}

\author{
ROBERTA E. DIHOFF \\ Rowan College of New Jersey, Glassboro, New Jersey \\ WILLIAM HETZNECKER \\ Temple University School of Medicine, Philadelphia, Pennsylvania \\ GARY M. BROSVIC \\ Rider College, Lawrenceville, New Jersey \\ and \\ LARA N. CARPENTER and LINDA S. HOFFMAN \\ Rowan College of New Jersey, Glassboro, New Jersey
}

\begin{abstract}
Ordinal diagnostic criteria were developed and used to assess the clinical characteristics of 20 autistic children. Subgroups of autistic children were identified and, on the basis of rankings on behavioral measures and performance on standardized tests and indices of school achievement, found to differ with respect to general level of functioning and educational placement. Developmental changes, expected in autistic children over a 12-year period, were reflected in higher rankings, especially for those in less restricted educational placements. Although the ordinal classification of autistic behavior is preliminary and the present results are based on the observation of only 20 children, the inclusion of ordinal criteria was found to promote high levels of diagnostic agreement among therapists and to facilitate the clinical monitoring of autistic children.
\end{abstract}

Beginning with the observations of Kanner (1943) and including the classification schemes of DSM I through DSM III-R, the autism diagnosis has been defined dichotomously through the presence or absence of symptoms. Autism, within the first and second DSM editions, was categorized as concurrent with childhood schizophrenia. Significant improvements have since been made in diagnostic criteria, and, in the DSM III-R, autism is now classified under the general rubric of pervasive developmental disorder (PDD). Fifty years of clinical research and practice have added substantially to the identification of salient symptomatology and behavioral indicators, relevant family history, and the course and prognosis of autism (Dahl, Cohen, \& Provence, 1986; Kolvin, 1971; Rutter, 1974; Rutter \& Schopler, 1987). Additional research is necessary, however, to broaden the autism diagnosis and to be able to indicate the degree of impairment and relate impairment to prognosis. However, the diagnosis of infantile autism remains difficult because of the presence of overlapping syndromes and disorders, as well as a lack of definitive criteria for establishing reliable diagnoses.

For example, Cohen, Paul, and Volkmar (1986) and Cohen, Volkmar, and Paul (1986) found that the criterion "pervasive lack of responsiveness" was too restrictive

Correspondence should be addressed to G. M. Brosvic, Biopsychology Laboratory, Department of Psychology, Rider College, 2083 Lawrenceville Rd., Lawrenceville, NJ 08648-3099. and did not account for the marked distinction between social awareness and responsiveness. Social avoidance is a diagnostic criterion; however, it is not clear whether such behavior reflects irreversible deficits caused by autism or a simple lack of effective reinforcing factors (Koegel, Dyer, \& Bell, 1987). Similarly, Beisler and Tsai (1987) observed that the use of gross language deficits, unusual speech patterns, and language delays as diagnostic criteria are subject to similar criticism.

In the psychiatric discipline more than in other areas of medicine, syndromes and disorders are not clearly delineated entities. The symptomatology of autism has been widely debated without the reaching of a clear consensus on diagnostic criteria and without the determination of the necessary and sufficient features for the diagnosis to be made. Recent attempts to achieve consensus have led to selected points of general agreement-for example, social relatedness and communication as core characteristics of the disorder-but not to the resolution of related issues of the definition and measurement of these characteristics and their developmental patterns. Similarly, issues pertaining to the prognostic value of differing levels of impairment on future functioning have not been resolved, and the application of DSM III-R criteria to autistic children in recent clinical studies has engendered the concept of a spectrum PDD and the possibility of subgroups within the larger PDD category (Wing \& Attwood, 1987).

For example, Cohen, Paul, and Volkmar (1986) and Cohen, Volkmar, and Paul (1986) proposed that Kanner's 
(1943) autism should be applied to only those individuals who not only meet DSM III-R criteria but also have an IQ ratio exceeding 70 . The spectrum of subgroups suggested by Cohen and associates includes autistic PPD, PDD, NOS (nor otherwise specified), and a new category, multiple PDD. The latter diagnosis is juxtaposed with PDD and specific DD; the major distinguishing feature is difficulty with regulative anxiety but not with social relatedness and cognitive dysfunction, as is typical with autistic or other PDD children (Hertzig, Snow, New, \& Shapiro, 1990). Similarly, Volkmar et al. (1987) reported subgroups of autistic children defined by Wing and Attwood's (1987) typology that did not match DSM III-R diagnostic criteria since both non-autistic and non-PDD children could be reliably subtyped with an apparent relationship emerging with the degree of mental retardation or with age and development associations. These subgroups suggest that the degree to which cognitive functioning is impaired is important for the autism diagnosis and that such impairment may be independent of the level of the autistic child's social development. Indeed, Hertzig et al. recently observed that DSM III-R criteria for the autism diagnosis suggest additional subgroups of autistic children that may include not only pervasive and socially unresponsive behaviors but also varying degrees of social impairment. This conclusion is similar to that in the neuropsychological studies of Fein, Pennington, Markowitz, Braverman, and Waterhouse (1986), in which sensorimotor and spatial development that function more effectively in autistic children have been reported to be less dependent on an intact capacity for affective expression, social interchange, and communication skills. Supporting the recurrence of the infantile autism deficit in social development as central in autistic PDD, Volkmer et al. distinguished autistic children from those diagnosed as mentally retarded and atypical PPD on the basis of ratings on the Revised Vineland Adaptive Behavior Scales. In practice, the evaluation of significantly impaired children on the basis of conventional means is difficult at best; therefore, additional measures of naturalistic behavior are needed. The three dimensions discriminating autistic children were significantly greater impairment in socialization, social development, and communication. Similarly, Wing and Attwood characterized three subtypes on the basis of clinical features: the aloof, the passive, and the active but odd.

In part, the seemingly disparate results described above are reflected in DSM III-R diagnostic criteria in which 8 of 16 items must be present, including a predetermined number from each of three major categories. Two major categories imply that some criteria are more applicable to the "younger or more handicapped" and others to the "older and less handicapped," suggesting both a range of severity for the disorder and a developmental pattern in its progression. Similarly, within each major category, there is a mixture of simple and complex behaviors. For example, in the no- or impaired-imitation category, behaviors range from a simple "wave good-bye" to "mechanical imitation of others' actions out of context," with the latter suggesting a more complex representation level. The issues surrounding the measurement of the varying degrees of severity of autistic children prompted development of an ordinal classification system for the evaluation and clinical monitoring of autistic children.

\section{METHOD}

\section{Subjects and Selection Criteria}

The subjects were 16 males and 4 females who were classified as autistic between the ages of 2 and 3 . Each child was diagnosed with autism before the age of 5 , which satisfied the DSM III criteria in use at the beginning of this study, and had reportedly demonstrated abnormalities before the age of $2 \frac{1}{2}$. Four children were African-American, 2 were Asian, and 1 was Hispanic. Three children were raised in bilingual homes. One child was diagnosed with hearing loss, 4 children had experienced seizures, and 2 children had neurological disorders. Two children had family histories of affective disorders.

\section{Classification System}

Following the formal diagnosis of autism, the clinical records of the autistic children were reviewed by staff psychiatrists and psychologists, and consensus was reached on five behavioral domains observed to be common to the children and on which varying levels of impairment could be operationally defined on at least an ordinal level of measurement: language ability, responsiveness to social situations, responsiveness to external physical stimulation, responsiveness to the environment, and adaptiveness demonstrated by functional use of materials. Level of $\operatorname{cog}$ nitive functioning served as the sixth domain.

Within each domain, five ordinal levels of functioning were operationalized and employed to rank-order clinical observations of the autistic children (see the Appendix). Ratings were independently performed by psychiatrists, clinical psychologists, and master's level psychology professionals. Median inter- and intrarater reliability exceeded $95 \%$, and the use of these ordinal criteria resulted in considerably higher levels of reliability in clinical monitoring than those achieved with the use of the DSM III criteria.

\section{Educational Environment}

One child participated in an experimental diagnostic classroom within a public school system, and another child was enrolled in a modified regular classroom setting with repetition of the grade. Two children attended specialized preschool settings. Seven children were in self-contained special education classes within public school systems, and the remaining 9 children participated in special schools for autistic children.

\section{Longitudinal Follow-up}

Four male and 3 female children from the sample described above were selected by the following criteria: at least 12 years between initial and longitudinal evaluations, normal sensory acuity (i.e., hearing), and absence of medical complications (i.e., seizures, abnormal EEG). The classification system described above was used, and the median interand intrarater reliability again exceeded $95 \%$.

\section{Statistical Analysis}

Principal components factor analysis with varimax orthogonal rotation was used to determine the statistical independence of the behavioral domains. Scales formed from the factor analysis were entered into a cluster analysis. Kruskal-Wallis tests were used to examine potential differences on each behavioral domain as a function of educational environment, and within two of these environments, potential differences in rankings on the behavioral domains were examined using the Friedman test, with each environment assigned an alpha of .01.

\section{RESULTS}

\section{Factor and Cluster Analyses}

As seen in Table 1, each of the five behavioral domains and the level of cognitive function loaded on a separate 
Table 1

Rotated Factor Loadings

\begin{tabular}{lcccccc}
\hline & Lang & Environ & Soc-Resp & Stim-Resp & Adapt & Cognit \\
\hline Lang & .88 & .10 & .21 & .26 & .31 & .09 \\
Environ & .08 & .95 & .12 & .14 & .03 & .23 \\
Soc-Resp & .19 & .14 & .93 & .09 & .15 & .22 \\
Stim-Resp & .23 & .15 & .09 & .93 & .25 & .05 \\
Adapt & .33 & .04 & .17 & .30 & .86 & .15 \\
Cognit & .11 & .40 & .36 & .07 & .19 & .78 \\
\hline
\end{tabular}

Note - Lang $=$ language ability, Environ $=$ responsiveness to the environment, Soc-Resp=social responsiveness, Stim-Resp=responsiveness to external stimuli, Adapt $=$ adaptability, and Cognit $=$ standing on appropriate developmental index.

factor, accounted for $83 \%$ of the explained variance, and formed single-item scales-language, environmental responsiveness, social responsiveness, stimulus response, adaptability, and cognition. Scores on the six scales were then entered into a cluster analysis from which two distinct dimensions emerged. The first dimension included environmental responsiveness, stimulus response, and cognition and was interpreted to represent general problemsolving ability. The second dimension included language, social responsiveness, and adaptability and was interpreted to represent general social awareness. Pearson productmoment correlations within the general problem-solvingability cluster were significant $(r=.88, p<.01)$, as were those within the general social awareness cluster $(r=.84, p<.01)$. Correlations between the items of the two clusters did not achieve statistical significance (all $r \mathrm{~s}<.15$, all $p s>.01$ ). Although the sample size was rather small, results of the correlational analyses confirm the relatedness of the items within, but not between, the general problem-solving and social awareness clusters.

\section{Educational Environment}

Children in more restricted educational environments (e.g., self-contained schools for autistic children) ranked lower on each behavioral domain than did children in less restricted educational environments [e.g., self-contained class in public school systems; Kruskal-Wallis tests, $t(4)=25.45, p<.01]$, and this finding supports the primacy of language and the ability to respond in appropriate ways to routine and novel situations.

In tests of the relative homogeneity of autistic children in two educational settings (7 in self-contained special education classes within public school systems, and 9 in special schools for autistic children), there were no significant differences in rankings across the six domains that were observed within each educational setting [Friedman tests, all $\chi^{2} s(5)<3.56$, all $\left.p s>.01\right]$. However, between these two educational settings, the children in the selfcontained special education classes within public school systems ranked higher on each domain than did those in special schools for autistic children [Mann-Whitney tests, all $t \mathrm{~s}(1)<20$, all $p \mathrm{~s}<.01]$.

\section{DISCUSSION}

The progression from a global, all-inclusive concept of a disorder to more specific delineated variants is common throughout medicine, psychology, and psychiatry. The present preliminary results, based on ordinal diagnostic criteria, support prior conclusions that autism is not a unitary construct. Indeed, the measurement system and resulting factors reported here are similar to the 15 scale items, scored from 1 to 4 and used to diagnose and evaluate the severity of autism, described previously by Schopler et al. (1988). Similarly, differences in cognitive level and social functioning implied by other authors were reflected in relatively homogeneous subgroups of autistic children; for example, children with high levels of social responsiveness and cognitive functioning (IQ > 70) required less restrictive educational placements and demonstrated greater improvement. As noted by Cohen, Paul, and Volkmar (1986) and Cohen, Volkmar, and Paul (1986), such children form a distinct subgroup within the spectrum of autism and confirm that autism is not a straightforward diagnosis. Indeed, the relative independence of the general social awareness and problem-solving-ability dimensions makes sense clinically and developmentally; however, additional studies with larger and more diverse samples must be conducted to confirm these preliminary findings.

The general social awareness dimension included social responsiveness, language ability, and adaptability, and the relatedness of these domains is indicative of the primacy of a social interactive deficit that determines the universe of autistic disorders. Just as autistic disorders themselves are distributed along one spectrum, so, too, are the phenomena that determine their range of expression or degree of manifestation. For example, autistic children often lack critically important capabilities such as paraverbal inflection, tone and volume of voice, eye contact and facial expression, and gesture and body posture, which characterize and determine the majority of social relatedness behaviors-for example, the receptive (the ability to "read" others) and the expressive (the ability to transmit nonsemantic nuances of affect and signals regarding interpersonal relatedness).

The factor identified as general problem-solving ability included environmental responsiveness, stimulus response, and cognition. Such a dimension is representative of impairments applicable to a broader spectrum of developmentally delayed children, including the mentally retarded, and this factor is prominent in autistic children and often manifest in more specific and discrete dysfunctions in children with specific developmental disorders. It should be noted, however, that mentally retarded children were not evaluated in the present study. Thus, a general model might posit that in the PDD group, the social deficit is primary whereas the deficit in general problem-solving ability is significant.

In some developmental disorders, social relatedness is either not impaired or, at most, minimally impaired; however, larger deficits in what we characterize as general problem solving are manifest and selectively effect the development of specific skills such as expressive language or the learning of highly sophisticated symbolic material (e.g., specific reading or numerical ability). The present results, although preliminary and based on a small sample of autistic children, call for expansion of the definition of language and representational abilities, such as the use of ordinal scales to denote levels of severity, critical to predicting the ability to adaptively function. In addition, operational definitions are needed to enhance the diagnostic process and to further delineate potential subgroups of autistic children. However, the identification of subgroups runs the risk of inadvertently establishing an invariate or fixed hierarchy of severity or disability that is projected prognostically and engenders a sense of futility or fatalism with respect to those children whose disorder may involve several dimensions of development and who rank lower in social awareness.

In summary, rank-order diagnostic criteria were found to facilitate the clinical monitoring of autistic children. Although a small sample was used, there was preliminary support for the hypothesis that there are relatively homogeneous subgroups of autistic children. The refinement of diagnostic criteria for autism, including ordinal measurement, will (1) enhance our knowledge and understanding of these disorders as global concepts are replaced with more refined and specific subtypes, 
(2) permit the placement of children into intervention programs specifically designed with respect to complexity, intensity, and appropriateness, and (3) enable the development and evaluation of appropriate long-term interventions specific to children with varying degrees of impairment in general problem solving and social awareness. The use of these criteria resulted in considerably higher levels of agreement between therapists, and additional studies are currently in progress examining the concurrent and predictive validity of the ordinal diagnostic criteria described above.

\section{REFERENCES}

Beisler, J. M., \& Tsai L. Y. (1987). Comparisons between autistic and nonautistic children on the test for auditory comprehension of language. Journal of Autism \& Developmental Disorders, 17, 95-101.

Cohen, D. J., Paul, R., \& Volkmar, F. R. (1986). Issues in the classification of pervasive developmental disorders: Toward DSM-IV. Journal of the American Academy of Child Psychiatry, 25, 213-220.

Cohen, D. J., Volkmar, F. R., \& Paul, R. (1986). Issues in the classification of pervasive developmental disorders: History and current status of nosology. Journal of the American Academy of Child Psychiatry, 25, 162-169.

Dahl, D. E., Cohen, D. J., \& Provence, S. (1986). Clinical and multivariate approaches to the nosology of pervasive developmental disorders. Journal of the American Academy of Child Psychiatry, 25, 170-180.

Fein, D., Pennington, B., Markowitz, P., Braverman, M., \& WATERHOUSE, L. (1986). Toward a neuropsychological model of infantile autism: Are the social deficits primary? Journal of the American Academy of Child Psychiatry, 25, 198-212.

Hertzig, M. E., SNow, M. E., New, E., \& Shapiro, T. (1990). DSM III and DSM III-R diagnosis of autism and pervasive developmental disorder in nursery school children. Journal of the American Academy of Child \& Adolescent Psychiatry, 29, 123-126.

KANNER, L. (1943). Autistic disturbances of affective contact. Nervous Child, 2, 217-250.

KoEgel, R. L., DYer, K., \& Bell, L. K. (1987). The influence of child-preferred activities on autistic children's social behavior. Journal of Applied Behavior Analysis, 20, 243-251.

Kolvin, I. (1971). Studies in childhood psychoses: I. Diagnostic criteria and classification. British Journal of Psychiatry, 118, 381-384.

RutTer, M. (1974). The development of infantile autism. Psychological Medicine, 4, 147-163.

RutTer, M., \& SCHOPLER, E. (1987). Autism and pervasive developmental disorders: Concepts and diagnostic issues. Journal of Autism \& Developmental Disorders, 17, 159-183.

Schopler, E., Reichsler, R. J., \& RenNer, B. R. (1988). Childhood Autism Rating Scale. Los Angeles: Western Psychological Services.

Volkmar, F. R., Sparrow, S. S., Goudreau, D., Cicchetti, D. V., PAul, R., \& CoHEN, D. J. (1987). Social deficits in autism: An operational approach using the Vineland adaptive behavior scales. Journal of the American Academy of Child \& Adolescent Psychiatry, 26, 156-161.
WING, L., \& ATTwood, A. (1987). Syndromes of autism and atypical development. In D. J. Cohen \& A. Donnelan (Eds.), Handbook of autism (pp. 145-165). New York: Wiley.

\section{APPENDIX \\ Ordinal Classification System}

Language

Gestures

Communicative intent

Communicative intent plus words (could include echolalia) Other-directed communication

Environmental Responsiveness

Bizarre responses

Oppositional behaviors

Acute spatial sense, may include preference for periphery Appropriate for brief periods

Appropriate responses

Social Responsiveness

Pervasive lack

Covert

Routine eye contact

Intermittent affective responses

Reciprocal social interchanges

Response to Physical Stimulation

No response

Increased sensitivity or stimulus hunger

Abnormal response in one dimension (i.e., pain or sound)

Appropriate response with delayed response time

Appropriate responses

Adaptiveness

Nonfunctional

Care for basic needs

Repetitive play

Appropriate isolated play

Representational play

Cognitive Level

Profound or severe

Moderate

Mild

Borderline

Normal

(Manuscript received February 27, 1993.) 